\title{
Multimedia data representation learning and applications
}

Published online: 4 January 2020

(C) Springer Science+Business Media, LLC, part of Springer Nature 2020

Multimedia Tools and Applications gratefully acknowledges the editorial work of the scholars listed below on the special issue entitled "Multimedia Data Representation Learning and Applications".

Of 33 papers submitted to this issue, 13 were eventually accepted after a stringent peerreview process.

\section{Corresponding Guest Editor}

\section{Xiaojun Chang}

Carnegie Mellon University, USA

Email: uqxchan1@cs.cmu.edu

\section{Guest Editors}

\section{Minnan Luo}

Xian Jiaotong University, China

Email: minnluo@xjtu.edu.cn

\section{Xiaodan Liang}

Carnegie Mellon University, USA

Email: xdliang328@gmail.com

\section{Yan Yan}

University of Michigan, USA

Email: yan@disi.unitn.it

Publisher's note Springer Nature remains neutral with regard to jurisdictional claims in published maps and institutional affiliations. 